10,11,05

\title{
Критические свойства в модели Изинга на треугольной решетке с переменным межслойным обменным взаимодействием
}

\author{
() А.К. Муртазаев, М.К. Рамазанов, М.К. Бадиев \\ Институт фризики ДагНЦ РАН, \\ Махачкала, Россия \\ E-mail: m_zagir@mail.ru
}

Поступила в Редакцию 6 мая 2019 г.

В окончательной редакции 22 мая 2019 г.

Принята к публикации 29 мая 2019 г.

\begin{abstract}
Репличным алгоритмом метода Монте-Карло выполнены исследования фазовых переходов, критических и термодинамических свойств трехмерной антиферромагнитной модели Изинга на слоистой треугольной решетке с переменным межслойным обменным взаимодействием. Исследования проведены для соотношений величины внутрислойного $J_{1}$ и межслойного $J_{2}$ обменных взаимодействий в диапазоне значений $r=J_{2} / J_{1}=0.01-1.0$. Установлено, что в рассмотренном интервале $r$ наблюдается фазовый переход второго рода. Используя теорию конечно-размерного скейлинга, рассчитаны статические критические индексы теплоемкости $\alpha$, восприимчивости $\gamma$, параметра порядка $\beta$, радиуса корреляции $v$, а также индекс Фишера $\eta$. Показано, что класс универсальности критического поведения этой модели сохраняется в интервале $0.05<r \leq 1.0$. Обнаружено, что при дальнейшем уменьшении величины $r$ наблюдается кроссовер от трехмерного критического поведения к квазидвумерному.
\end{abstract}

Ключевые слова: фрустрации, метод Монте-Карло, модель Изинга, фазовый переход.

DOI: 10.21883/FTT.2019.10.48267.469

\section{1. Введение}

Исследование фазовых переходов (ФП) и критических явлений фрустрированных решеточных спиновых систем является одной их актуальных задач физики конденсированного состояния $[1,2]$.

Фрустрированные системы во многом проявляют свойства, отличные от соответствующих нефрустрированных систем. Это отличие выражается в богатом разнообразии фаз и ФП, что обусловлено сильным вырождением и высокой чувствительностью таких систем к различного рода возмущающим взаимодействиям. Поэтому в настоящее время продолжаются интенсивные исследования природы ФП, тепловых, магнитных и критических свойств фрустрированных спиновых систем [3-10].

Модель Изинга на треугольной решетке с антиферромагнитным взаимодействием первых ближайших соседей разупорядочена при нулевой температуре, нарушая третий закон термодинамики [8]. Взаимодействие вторых ближайших соседей играет решающую роль, вызывая различные виды дальнего порядка в зависимости от знака взаимодействия вторых ближайших соседей.

Теоретические и численные исследования трехмерной антиферромагнитной модели Изинга на слоистой треугольной решетке в значительной степени согласуются с критическими свойствами, характеризующие высокотемпературным ФП. Однако результаты теоретических исследований, полученные ранее, оказались спорными. Теория Ландау-Гинзбурга-Вильсона предложила 3D $X Y$-поведение для наблюдаемого магнитного ФП [11]. Позже, результаты моделирования метода
Монте-Карло (МК) дали результаты, характерные для трикритического поведения [12]. Более поздние исследования с использованием гистограммного метода МК и теории конечно-размерного скейлинга (КРС), получили набор критических индексов, которые отличаются от трикритического поведения и опять-таки довольно близкие к 3D $X Y$-модели [13-15].

Исследование магнитных материалов с фрустрациями в большинстве случаев проводится численными методами. Это связано с тем, что экспериментальные и теоретические методы сталкиваются с серьезными проблемами при исследовании ФП и критических свойств таких материалов. Несмотря на это, в литературе встречаются работы, посвященные экспериментальному исследованию эффектов фрустрации в соединениях, которые могут быть описаны в рамках рассматриваемой нами модели. $\mathrm{B}$ работе [16] изучены порошковые соединения $\mathrm{VX}_{2}$, $\mathrm{X} \equiv \mathrm{Cl}, \mathrm{Br}$ и $\mathrm{I}$, которые представляют собой квазидвумерные треугольные антиферромагнетики. Спины в соединениях $\mathrm{VCl}_{2}, \mathrm{VBr}_{2}$ и $\mathrm{VI}_{2}$, как было обнаружено, упорядочены при температурах $36.0 \mathrm{~K}, 29.5 \mathrm{~K}$ и $16.3 \mathrm{~K}$ соответственно, образуя структуру трех подрешеток. $\mathrm{B}$ соединениях $\mathrm{VCl}_{2}$ и $\mathrm{VBr}_{2}$ сильное диффузное рассеяние может наблюдаться не только при $T>T_{N}$, но и при $T \ll T_{N}$. Критической характеристики рассеяния для двумерного случая не видно, но изменение температуры подрешеточной намагниченности довольно близко к таковому для системы Изинга.

В работе [17] авторами изучены упорядоченные фазы и ФП в модели Изинга на слоистой треугольной решетке с сильным межплоскостным взаимодействием. 
Эта модель может быть использована для описания соединений $\mathrm{CsCoCl}_{3}$ и $\mathrm{CsCoBr}_{3}$. Авторы обнаружили, что в промежуточном состоянии одна из трех магнитных подрешеток частично не упорядочена. Низкотемпературная фаза этих соединений представляет собой, так называемую двухподрешеточную ферримагнитную фазу. Изучая распределения доменных стенок в одномерных цепях, соединяющих слоистые треугольные решетки, в работе так же изучена природа ФП.

Результаты, полученные для этой модели в рамках приближения среднего поля [18], а также кластерным вариационным методом [19], показывают, что в промежуточном состоянии одна из трех магнитных подрешеток не упорядочена.

Вместе с тем, результаты метода МК показывают, что такие частично неупорядоченные состояния описываются модой со случайно изменяющейся в пространстве и во времени фазой [20-22].

Результаты МК моделирования изинговского антиферромагнетика с учетом антиферромагнитных взаимодействий вторых ближайших соседей указывают на существование ФП первого порядка между низкотемпературной двухподрешеточной антиферромагнитной фазой и высокотемпературной парамагнитной фазой. Этот сценарий отличается от того, который наблюдается, когда взаимодействия первых ближайших соседей являются ферромагнитным. Для такого случая, фаза Березинского-Костерлица-Таулеса возникает при промежуточной температуре между трехподрешеточной ферримагнитной фазой при низкой температуре и парамагнитной фазой при высокой температуре [23].

На сегодняшний день практически не изучены вопросы, связанные с влиянием величины межслойного обменного взаимодействия на природу ФП и критические свойства изинговских антиферромагнетиков. В связи с этим, в настоящей работе нами предпринята попытка исследовать природу ФП, критические и термодинамические свойства антиферромагнитной модели Изинга на слоистой треугольной решетке с переменным межслойным обменным взаимодействием.

Исследование данной модели на основе современных методов и идей позволит получить ответ на ряд вопросов, связанных с критическим поведением слоистых фрустрированных спиновых систем.

\section{2. Модель и метод исследования}

Гамильтониан антиферромагнитной трехмерной модели Изинга на треугольной решетке может быть представлен в следующем виде:

$$
H=-J_{1} \sum_{\langle i, j\rangle}\left(S_{i} \cdot S_{j}\right)-J_{2} \sum_{\langle i, j\rangle}\left(S_{i} \cdot S_{l}\right)-J_{3} \sum_{\langle i, j\rangle}\left(S_{i} \cdot S_{k}\right),
$$

где $S_{i, l, k}= \pm 1-$ изинговский спин, $J_{1}<0, J_{2}<0$ и $J_{3}>0$ - константы обменного взаимодействия. Решетка состоит из двумерных треугольных слоев, сложенных по ортогональной оси. Первый член в формуле (1) характеризует антиферромагнитное взаимодействие всех ближайших соседей, которое берется одинаковой внутри слоя, второй член характеризует межслойное антиферромагнитное взаимодействие и третий член характеризует ферромагнитное взаимодействие вторых ближайших соседей внутри слоев. При расчете, мы принимаем, что $\left|J_{1}\right|=\left|J_{3}\right|=1$ и меняем значение $J_{2} \cdot r=J_{2} / J_{1}-$ соотношение межслойного и внутрислойного обменов. Bсе параметры приводятся в безразмерных величинах. В настоящей работе рассматривается интервал $0.01<r \leq 1.0$.

Данная модель позволяет описать результаты эксперимента различных систем, включая искусственные дипольные магниты [24], такие материалы, как $\mathrm{Ba}_{3} \mathrm{CuSb}_{2} \mathrm{O}_{9}$, где электрически заряженные гантели действуют как изинговские степени свободы [25], фрустрированные кулоновские жидкости [26] и др.

В последние годы такие системы на основе микроскопических гамильтонианов успешно изучаются методами МК [27-33]. В последнее время разработано много новых вариантов алгоритмов метода МК. Одними из наиболее эффективных для исследования подобных систем является репличный обменный алгоритм метода МК [30,31]. Поэтому нами, в данном исследовании был использован этот алгоритм.

\section{3. Результаты моделирования}

Расчеты проводили для систем с периодическими граничными условиями и линейными размерами $L \times L \times L=N, L=18-48$, где $L-$ измеряется в размерах элементарной ячейки. Для вывода системы в состояние термодинамического равновесия отсекается участок длиной $\tau_{0}=4 \times 10^{5} \mathrm{MKsteps} / \mathrm{spin}$, что в несколько раз больше длины неравновесного участка. Усреднение термодинамических величин выполняли вдоль марковской цепи длиной $\tau=500 \tau_{0}$ MKsteps/spin.

На рис. 1 показана магнитная структура основного состояния исследуемой модели, полученная для соседних слоев. Здесь показано, что основное состояние может иметь шестикратное вырождение. Структура упорядоченной фазы образована чередованием рисунков, показанных на рис. 1.

Параметр порядка системы m вычислялся, используя выражение [27]:

$$
m=\frac{3}{N} \sqrt{\left\langle M_{A}^{2}+M_{B}^{2}+M_{C}^{2}\right\rangle / 3},
$$

где $M_{A}, M_{B}$ и $M_{C}$ - намагниченности трех подрешеток.

Для наблюдения за температурным ходом теплоемкости и восприимчивости использованы выражения [27]:

$$
\begin{gathered}
C=\left(N K^{2}\right)\left(\left\langle U^{2}\right\rangle-\langle U\rangle^{2}\right), \\
\chi= \begin{cases}(N K)\left(\left\langle m^{2}\right\rangle-\langle|m|\rangle^{2}\right), & T<T_{N} \\
(N K)\left\langle m^{2}\right\rangle, & T \geq T_{N}\end{cases}
\end{gathered}
$$

где $K=\left|J_{1}\right| / k_{\mathrm{B}} T, N-$ число частиц, $T_{N}-$ критическая температура, $U$ - внутренняя энергия, $m$ - параметр порядка ( $U$ и $m$ являются нормированными величинами). 

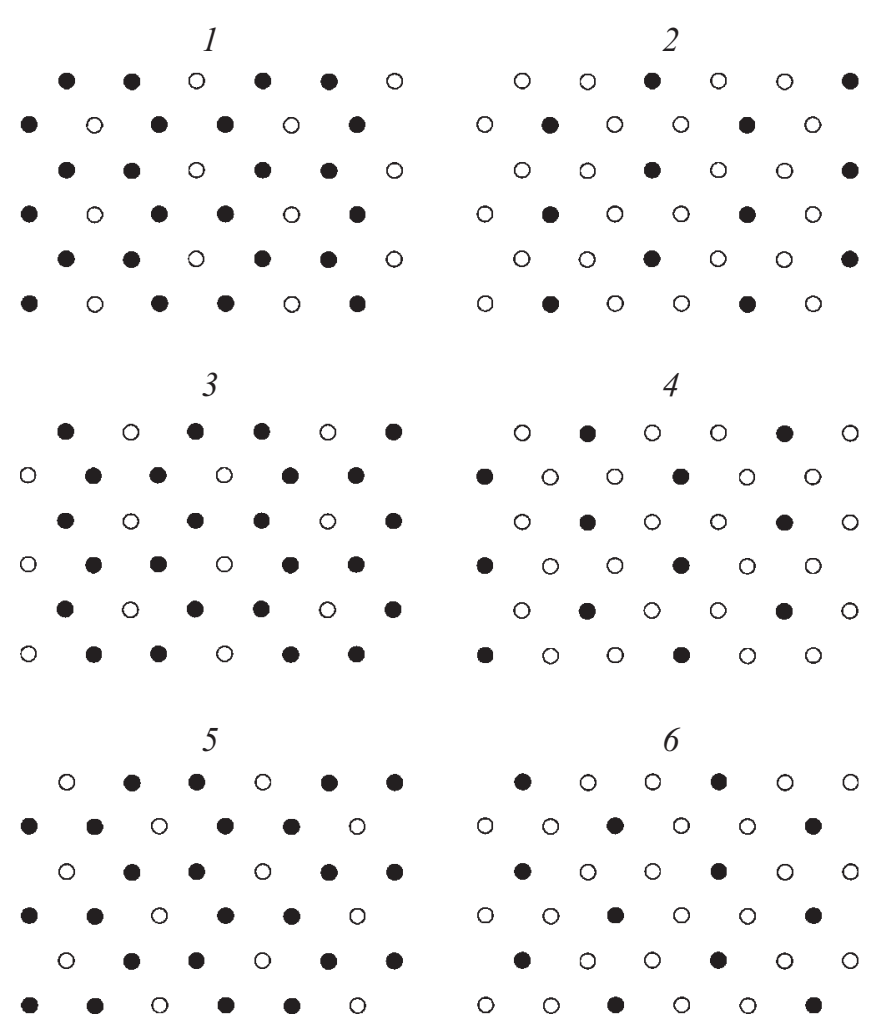

Рис. 1. Магнитная структура основного состояния.

На рис. 2 и рис. 3 представлены температурные зависимости теплоемкости и восприимчивости, полученные при разных $L$ для значения $r=0.8$ (здесь и далее статистическая погрешность не превышает размера символов, использованных для построения зависимостей). Из рисунков видно, что на кривых зависимости теплоемкости и восприимчивости от температуры для всех значений $L$ вблизи критической температуры наблюдаются хорошо выраженные максимумы, которые увеличиваются с ростом числа спинов в системе, причем эти максимумы в пределах погрешности приходятся на одну и ту же температуру.

Для анализа характера ФП, особенностей поведения тепловых характеристик вблизи критической точки и определения критической температуры $T_{N}$ наиболее эффективным является метод кумулянтов Биндера четвертого порядка, который имеет вид [34]:

$$
U_{L}=1-\frac{\left\langle m^{4}\right\rangle_{L}}{3\left\langle m^{2}\right\rangle_{L}^{2}},
$$

Согласно теории КРС, точка пересечения всех кривых $U_{L}(T)$ является критической точкой. Выражение (5) позволяет определить критическую температуру $T_{N} \mathrm{c}$ большой точностью. В случае ФП второго рода кривые температурной зависимости кумулянтов Биндера $U_{L}$ имеют четко выраженную точку пересечения [34]. Следует отметить, что применение метода кумулянтов Бин- дера позволяет также хорошо тестировать тип ФП в системе.

На рис. 4 представлены характерные зависимости $U_{L}$ от температуры при $r=0.8$ для разных значений $L$. Видно, что в критической области наблюдается четко выраженная точка пересечения $\left(T_{N}=8.031(3)\right.$; здесь и далее температура дана в единицах $\left.\left|J_{1}\right| / k_{\mathrm{B}}\right)$. Аналогичным образом были определены критические температуры и для остальных значений $r$ которые приведены в таблице.

Для анализа рода ФП нами использовался гистограммный анализ данных метода МК $[35,36]$. Этот метод позволяет надежно определить род ФП.

Результаты нашей работы показывают, что ФП для всех рассмотренных нами значений $r$ являются переходами второго рода. Это продемонстрировано на рис. 5, где представлена гистограмма распределения

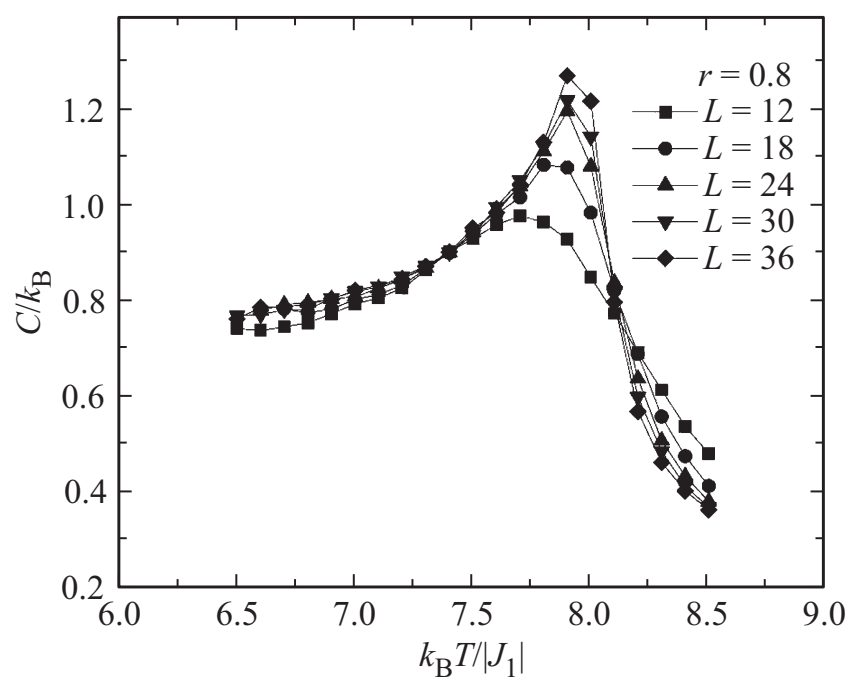

Рис. 2. Зависимость теплоемкости $C / k_{\mathrm{B}}$ от температуры $k_{\mathrm{B}} T /\left|J_{1}\right|$ для $r=0.8$.

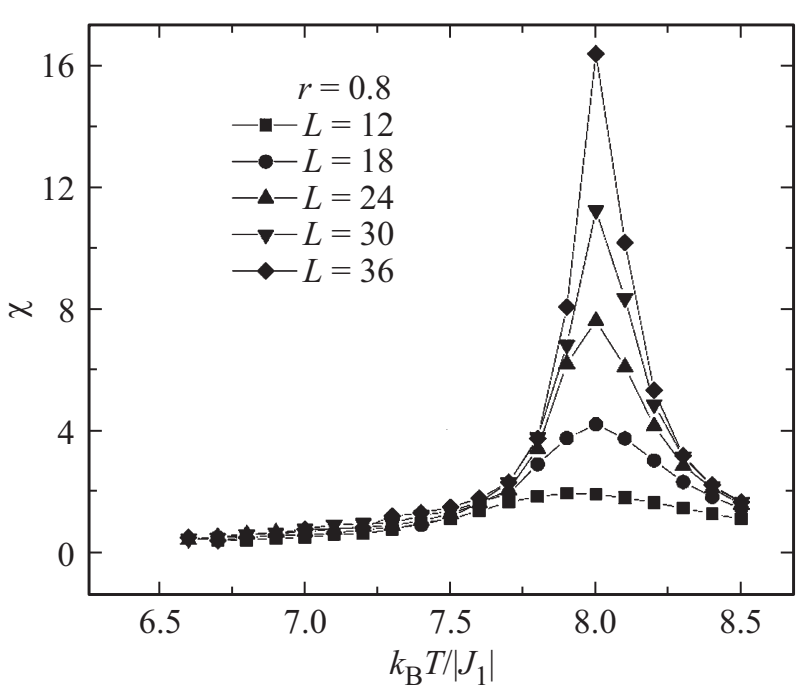

Рис. 3. Зависимость теплоемкости $\chi$ от температуры $k_{\mathrm{B}} T /\left|J_{1}\right|$ для $r=0.8$. 
Значения критических индексов для модели Изинга на слоистой треугольной решетке с переменным межслойным обменным взаимодействием

\begin{tabular}{l|l|l|l|l|l|l|c}
\hline$r$ & $T_{N}$ & $v$ & $\alpha$ & $\beta$ & $\gamma$ & $\eta$ & $\alpha+2 \beta+\gamma=2$ \\
\hline 0.01 & $5.420(3)$ & $0.577(15)$ & $0.26(5)$ & $0.284(15)$ & $0.81(5)$ & $059(10)$ & 1.6 \\
0.05 & $5.751(3)$ & $0.592(15)$ & $0.22(5)$ & $0.292(15)$ & $0.88(5)$ & $0.51(10)$ & 1.6 \\
0.1 & $6.066(3)$ & $0.651(15)$ & $0.05(5)$ & $0.370(15)$ & $1.19(5)$ & $0.16(10)$ & 1.98 \\
0.2 & $6.465(3)$ & $0.648(15)$ & $0.05(5)$ & $0.365(15)$ & $1.21(5)$ & $0.13(10)$ & 1.99 \\
0.4 & $7.087(3)$ & $0.660(15)$ & $0.02(5)$ & $0.369(15)$ & $1.20(5)$ & $0.18(10)$ & 1.95 \\
0.6 & $7.5850(3)$ & $0.648(15)$ & $0.07(5)$ & $0.362(15)$ & $1.17(5)$ & $0.19(10)$ & 1.96 \\
0.8 & $8.031(3)$ & $0.652(15)$ & $0.05(5)$ & $0.373(15)$ & $1.20(5)$ & $0.15(10)$ & 1.99 \\
1.0 & $8.427(3)$ & $0.650(15)$ & $0.05(5)$ & $0.370(15)$ & $1.16(5)$ & $0.21(10)$ & \\
{$[33]$} & & $0.669(7)$ & $-0.01(2)$ & $0.345(12)$ & $1.316(9)$ & &
\end{tabular}

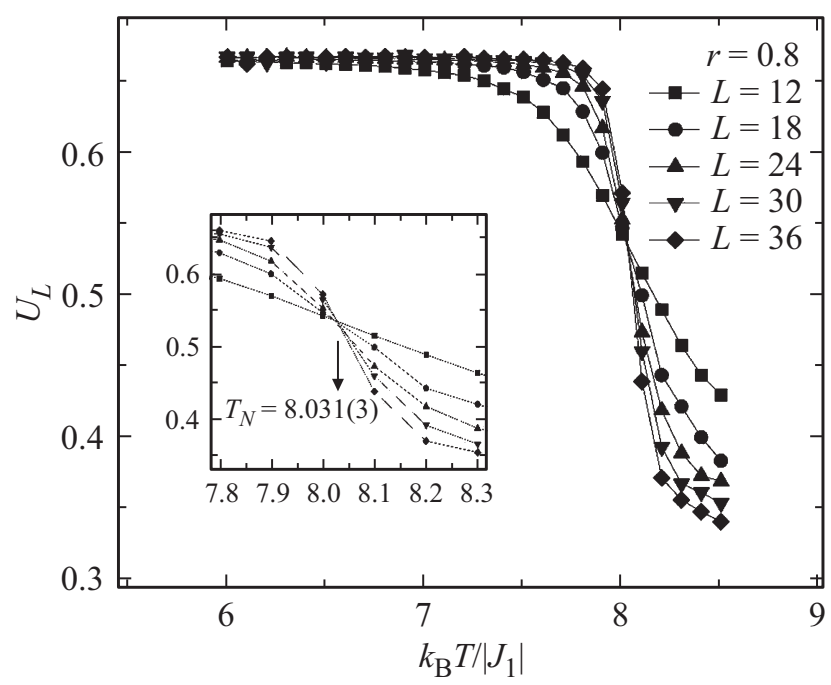

Рис. 4. Зависимости кумулянта Биндера $U_{L}$ от температуры $k_{\mathrm{B}} T /\left|J_{1}\right|$ для $r=0.8$ при различных $L$.

энергии для системы с линейными размерами $L=90$ при $r=0.8)$. Из представленной гистограммы следует, что в зависимости вероятности $P$ от энергии $U$ наблюдается один хорошо выраженный максимум, который свидетельствует в пользу ФП второго рода. Аналогичный результат получен и для всех остальных значений $r$.

Для расчета статических критических индексов теплоемкости $\alpha$, восприимчивости $\gamma$, параметра порядка $\beta$, радиуса корреляции $v$ и индекса Фишера $\eta$ применялись соотношения теории КРС [32].

Из теории КРС следует, что в системе с размерами $L \times L \times L$ при $=T_{N}$ и достаточно больших $L$ выполняются следующие выражения:

$$
\begin{aligned}
m & \sim L^{-\beta / v}, \\
\chi & \sim L^{\gamma / v}, \\
V_{n} & \sim L^{1 / v} g_{V_{n}},
\end{aligned}
$$

где $g_{V_{n}}$ - некоторая постоянная, а в качестве $V_{n}$ могут выступать

$$
V_{n}=\frac{\left\langle m^{n} U\right\rangle}{\left\langle m^{n}\right\rangle}-\langle U\rangle, \quad(n=1,2,3)
$$

Эти выражения были нами использованы для определения $\beta, \gamma$ и $v$.

Для аппроксимации температурной зависимости теплоемкости от $L$ на практике, как правило, используется выражение

$$
C_{\max }(L)=A_{1}-A_{2} L^{\alpha / v},
$$

где $A_{1}$ и $A_{2}-$ некоторые коэффициенты.

На рис. 6 в двойном логарифмическом масштабе представлены характерные зависимости параметров $V_{n}$ при $n=1,2,3$ от линейных размеров решетки $L$ для $r=0.8$. Как видно из рисунка все точки на графиках в пределах погрешности хорошо ложатся на прямые. Зависимости на рисунках, проведенные в соответствии с методом наименьших квадратов, параллельны друг другу. Углы наклона прямых определяют значения $1 / v$. Вычисленные таким образом значения $v$ использовались

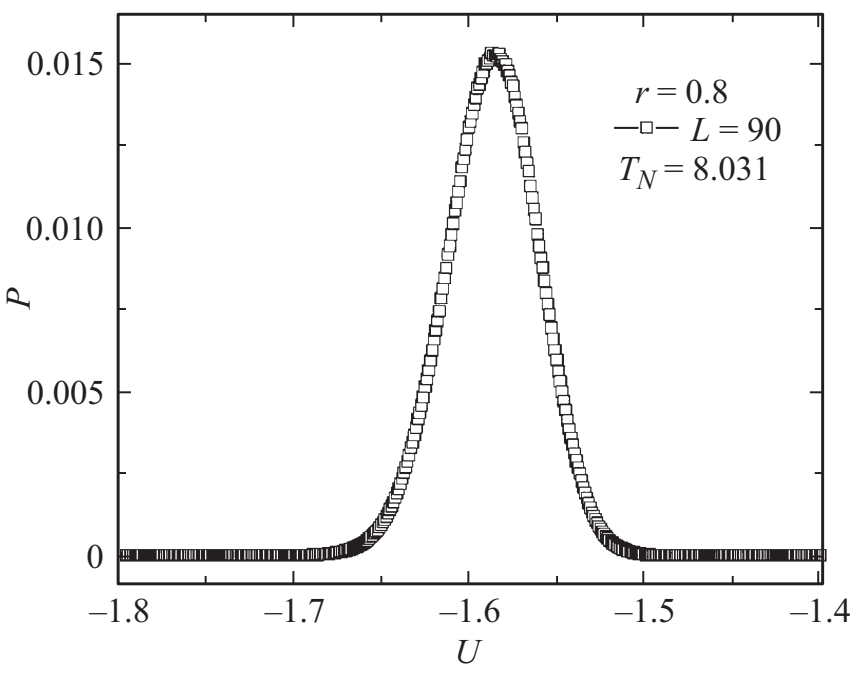

Рис. 5. Гистограмма распределения энергии для $r=0.8$ при $L=90$. 


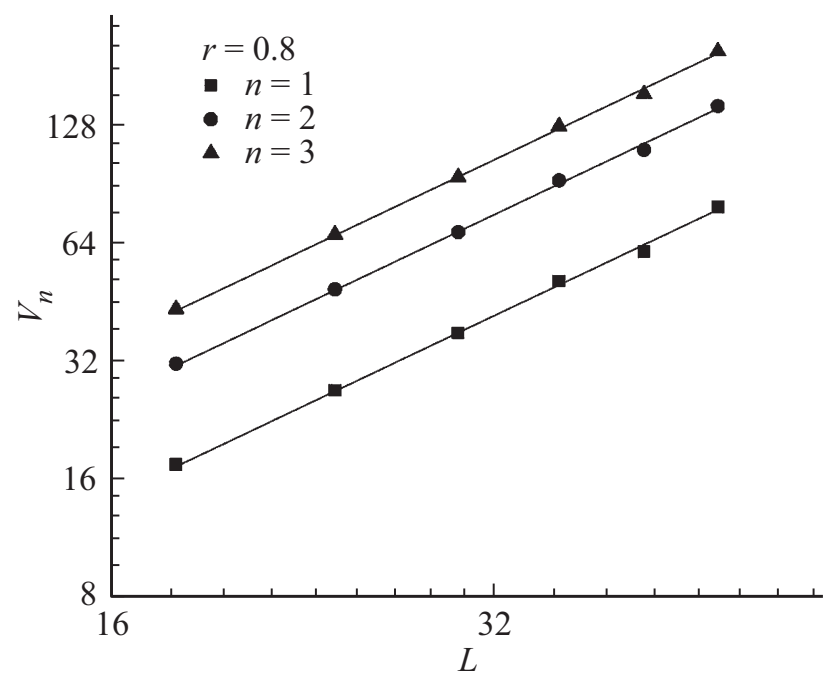

Рис. 6. Зависимость параметра $V_{n}$ от линейных размеров системы $L$ при $T=T_{N}$ для $r=0.8$.

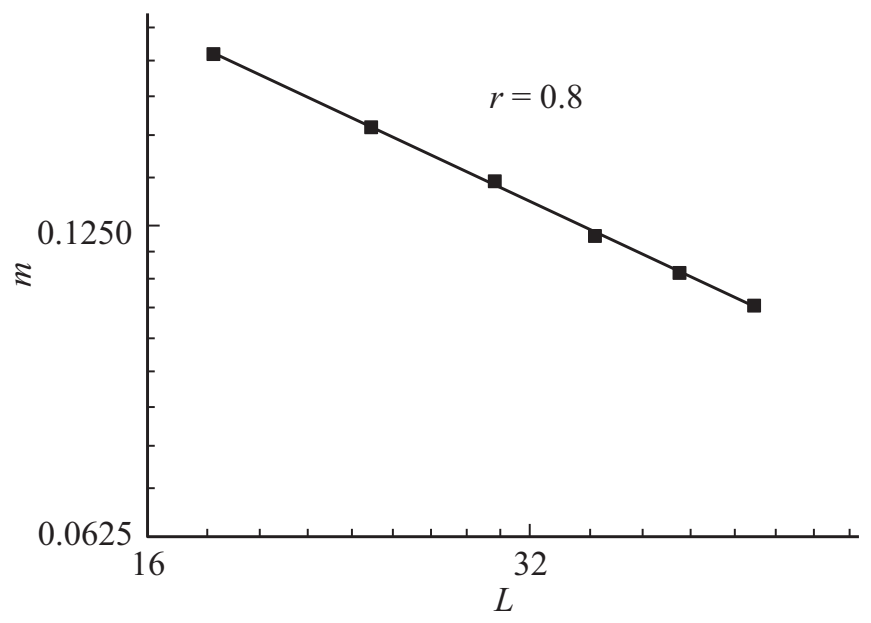

Рис. 7. Зависимость параметра порядка $m$ от линейных размеров системы $L$ при $T=T_{N}$ для $r=0.8$.

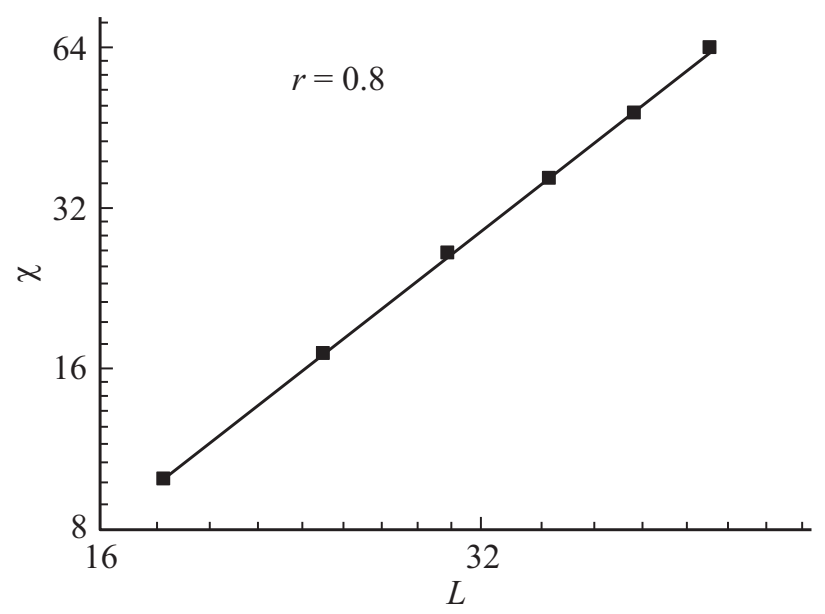

Рис. 8. Зависимость восприимчивости $\chi$ от линейных размеров системы $L$ при $T=T_{N}$ для $r=0.8$. для определения критических индексов теплоемкости $\alpha$, восприимчивости $\gamma$ и параметра порядка $\beta$.

На рис. 7 и 8 в двойном логарифмическом масштабе представлены характерные зависимости магнитного параметра порядка $m$ и восприимчивости $\chi$ от линейных размеров решетки $L$ для $r=0.8$. Все точки в пределах погрешности ложатся на прямые. Углы наклона этих прямых определяют значения $\beta / v$ и $\gamma / v$. По этой схеме были определены значения и для теплоемкости $\alpha / v$. Имея данные по $v$, вычислялись статические критические индексы $\alpha, \beta$ и $\gamma$.

Эта процедура использовалась для расчета критических индексов для всех рассмотренных значений $r$. Все значения статических критических индексов, полученные в данной работе, представлены в таблице.

Как видно из таблицы численные значения критических индексов в интервале $0.05<r \leq 1.0$ в пределах погрешности совпадают между собой. Отметим так же, что с достаточно высокой точностью выполняются скейлинговые соотношение между критическими индексами. При этом значения критических индексов в интервале $0.05<r \leq 1.0$ не зависят от величины межслойных взаимодействий и в пределах погрешности совпадают со значениями критических индексов трехмерной $X Y$ модели [37], что свидетельствует о принадлежности данной модели к $X Y$-классу универсальности критического поведения. При $r \leq 0.05$ значения критических индексов существенно меняются и уже не выполняются скейлинговые соотношения между ними. Можно предположить, что при значении $r=0.05$ в системе происходит кроссовер от трехмерного критического поведения к квазидвумерному.

\section{4. Заключение}

Исследование фазовых переходов и критических свойств трехмерной антиферромагнитной модели Изинга на слоистой треугольной решетке с переменным межслойным обменным взаимодействием выполнено с использованием высокоэффективного репличного алгоритма метода Монте-Карло. На основе гистограммного метода анализа данных и метода кумулянтов Биндера четвертого порядка проведен анализ характера фазовых переходов. Показано, что в исследуемой модели реализуется фазовый переход второго рода. Рассчитаны все основные статические критические индексы. Полученные данные свидетельствуют о принадлежности трехмерной модели Изинга на слоистой треугольной решетке к $X Y$-классу универсальности критического поведения. Обнаружено, что класс универсальности критического поведения этой модели сохраняется вплоть до значения межслойного обменного взаимодействия $r>0.05$, и при дальнейшем уменьшении величины $r$ наблюдается переход от трехмерного критического поведения к квазидвумерному. 


\section{Финансирование работы}

Исследование выполнено при финансовой поддержке РФФИ в рамках научных проектов № 19-02-00153-а, 18-32-20098-мол-а-вед и № 18-32-00391-мол-а.

\section{Конфликт интересов}

Авторы заявляют, что у них нет конфликта интересов.

\section{Список литературы}

[1] A.Z. Patashinskii, V.L. Pokrovskii. Fluctuation Theory of Phase Transitions. Pergamon, Oxford (1979).

[2] Sh. Ma. Modern Theory of Critical Phenomena. SpringerVerlag, London (1976).

[3] R.S. Gekht. Usp. Fiz. Nauk 32, 871 (1989).

[4] K. Katsumata, H. Aruga Katori, S. Kimura, Y. Narumi, M. Hagiwara, K. Kindo. Phys. Rev. B 82, 104402 (2010).

[5] E.H. Boubcheur, H.T. Diep. Phys. Rev. B 58, 5163 (1998).

[6] S.E. Korshunov. Phys. Rev. B 72144417 (2005).

[7] E.Z. Ising. Phys. 31, 253 (1924).

[8] G.H. Wannier. Phys. Rev. 79, 357 (1950).

[9] G.F. Newell. Phys. Rev. 79, 876 (1950).

[10] R.M.F. Houtapel. Physica 16, 425 (1950).

[11] G.A. Baker, Jr., B.G. Nickel, D.I. Meiron. Phys. Rev. B 17, 1365 (1978).

[12] O. Heinonen, R.G. Petschek. Phys. Rev. B 40, 9052 (1989).

[13] M.L. Plumer, A. Mailhot, R. Ducharme, A. Caille, H.T. Diep. Phys. Rev. B 47, 14312 (1993).

[14] A. Bunker, B.D. Gaulin, C. Kallin. Phys. Rev. B 48, 15, 861 (1993).

[15] N. Todoroki, S. Miyashita. J. Phys. Soc. Jpn. 73, 412 (2004).

[16] K. Hirakawa, H. Kadowaki, K.Ubukoshi. J. Phys. Soc. Jpn. 52, 1814 (1983).

[17] N. Todoroki, Z. Miyashita. J. Phys. Soc. Jpn. 73, 412 (2004).

[18] H. Shiba. Prog. Teor. Phys. 64, 466 (1980).

[19] M. Kaburagi, T. Tonegawa, J. Kanamori. J. Phys. Soc. Jpn. 51, 3857 (1982).

[20] P. Matsubara, S. Ikeda. Phys. Rev. B 28, 4064 (1983).

[21] P. Matsubara, S. Inawashira. J. Phys. Soc. Jpn. 53, 4373 (1984).

[22] H. Yoshizawa, K. Hirakawa. J. Phys. Soc. Jpn. 46, 448 (1980).

[23] E. Rastelli, S. Regina, A. Tassi. Phys. Rev. 71, 174406 (2005).

[24] E. Mengotti, L.J. Heyderman, A. Bisig, A. Fraile Rodríguez, L. Le Guyader, F. Nolting, H.B. Braun. J. Appl. Phys. 105, 113113 (2009).

[25] A. Smerald, F. Mila. Phys. Rev. Lett. 115, 147202 (2015).

[26] S. Mahmoudian, L. Rademaker, A. Ralko, S. Fratini, V. Dobrosavljević. Phys. Rev. Lett. 115, 025701 (2015).

[27] A.K. Murtazaev, M.K. Ramazanov, M.K. Badiev. Physica A 507, 210 (2018).

[28] А.К. Муртазаев, М.К. Рамазанов, Д.Р. Курбанова, М.К. Бадиев, Я.К. Абуев. ФТТ 59, 1082 (2017).

[29] M.K. Ramazanov, A.K. Murtazaev, M.A. Magomedov. Physica A 521, 543 (2019).

[30] А.К. Муртазаев, М.К. Рамазанов, Д.Р. Курбанова, М.А. Магомедов, М.К. Бадиев, М.К. Мазагаева. ФТТ 61, 1170 (2019).

[31] М.К. Бадиев, А.К. Муртазаев, М.К. Рамазанов. ЖЭТФ 150, 722 (2016).
[32] A.K. Murtazaev, M.K. Ramazanov, M.K. Badiev. Physica B 476, 1 (2015).

[33] M.K. Ramazanov, A.K. Murtazaev, M.A. Magomedov, M.K. Badiev. Phase Transitions 91, 610 (2018).

[34] К. Биндер, Д.В. Хеерман. Моделирование методом МонтеКарло в статистической физике. Наука, М. (1995).

[35] F. Wang, D.P. Landau. Phys. Rev. Lett. 86, 2050 (2001).

[36] М.К. Рамазанов, А.К. Муртазаев. Письма в ЖЭТФ 106, 72 (2017).

[37] J.C. Le Guillou, J. Zinn-Justin. J. Phys. Lett. 46, 137 (1985).

[38] M.F. Collins. Magnetic critical scattering. Oxford University Press, Oxford (1989).

Редактор Т.Н. Василевская 